\title{
Os recursos fisioterapêuticos na reabilitação de mulheres pós mastectomizadas
}

\author{
Physiotherapeutic resources in the rehabilitation of post mastectomized women
}

Recursos fisioterapéuticos en la rehabilitación de mujeres post mastectomizadas

Lília Tatiane dos Santos Sá1*, Carla Lorena de Araújo Costa ${ }^{1}$, Marcio Santos da Conceição¹, Midiã Oliveira Lima1, Carolline Bittencourt da Cruz¹, Raiane Santos de Brito', Luana de Jesus Reis'.

\begin{abstract}
RESUMO
Objetivo: Discutir de que forma os recursos fisioterapêuticos contribuem na reabilitação de mulheres pós mastectomizadas. Métodos: Revisão de literatura no período de 2009 a 2019 nas bases de dados BVS, PubMed, SciELO, PEDro, e Google Acadêmico. O processo de análise do artigo selecionado deu-se por meio da leitura exploratória e detalhada de títulos, resumos e dos resultados das pesquisas, onde se buscaram os recursos fisioterapêuticos aplicados na reabilitação de mulheres pós mastectomizadas. Resultados: Dentre os recursos fisioterapêuticos os mais utilizados destacam-se exercícios livres, alongamentos, massoterapia, mobilização cicatricial e pompagem, onde estes têm o intuito de reduzir os impactos das disfunções advindas da cirurgia que influência diretamente na funcionalidade e a qualidade de vida da paciente pós mastectomizada. Considerações Finais: Foi possível observar que os recursos fisioterapêuticos, proporcionaram resultados positivos na redução da algia, na manutenção e ganho de ADM e no aumento da força muscular de mulheres submetidas à mastectomia.
\end{abstract}

Palavras-chave: Fisioterapia, Mastectomia, Saúde da mulher.

\begin{abstract}
Objective: Discuss how physical therapy resources contribute to the rehabilitation of post-mastectomized women. Methods: Literature review from 2009 to 2019 in the databases VHL, PubMed, SciELO, PEDro, and Google Scholar. The analysis process of the selected article took place through the exploratory and detailed reading of titles, summaries and research results, where the physiotherapeutic resources applied in the rehabilitation of post-mastectomized women were sought. Results: Among the most used physiotherapeutic resources are free exercises, stretching, massage therapy, scar mobilization and pomp. Results: Among the physiotherapeutic resources the most used are free exercises, stretching, massage therapy, scar mobilization and pomp, where these aim to reduce the impacts of dysfunctions arising from surgery that directly influences the functionality and quality of life of the post-mastectomized patient. Final Conclusions: It was observed that the physiotherapeutic resources provided positive results in the reduction of pain, maintenance and gain of ROM and increase of muscle strength in women undergoing mastectomy.
\end{abstract}

Keywords: Physiotherapy, Mastectomy, Women's health.

\section{RESUMEN}

Objetivo: Discuta cómo los recursos de fisioterapia contribuyen a la rehabilitación de mujeres post mastectomizadas. Métodos: Revisión de literatura de 2009 a 2019 en las bases de datos VHL, PubMed, SciELO, PEDro y Google Scholar. El proceso de análisis del artículo seleccionado se llevó a cabo mediante la lectura exploratoria y detallada de títulos, resúmenes y resultados de investigación, que buscaban los recursos fisioterapéuticos aplicados en la rehabilitación de mujeres posmastectomizadas. Resultados: Entre los recursos de fisioterapia, los más utilizados son ejercicios gratuitos, estiramientos, terapia de masajes, movilización de cicatrices y pompa, donde estos tienen como objetivo reducir los impactos de las

${ }^{1}$ Centro Universitário UNIRB (FARAL), Alagoinhas - BA. *E-mail: thatty-sa@hotmail.com 
disfunciones derivadas de la cirugía que influye directamente en la funcionalidad y la calidad de vida del paciente posmastectomizado. Conclusiones Finales: Se observó que los recursos fisioterapéuticos proporcionaron resultados positivos en la reducción del dolor, el mantenimiento y la ganancia de ROM y el aumento de la fuerza muscular de las mujeres sometidas a mastectomía.

Palabras clave: Fisioterapia, Mastectomía, Salud de la mujer.

\section{INTRODUÇÃO}

O Instituto Nacional do Câncer (2014) define o carcinoma mamário como uma doença resultante da multiplicação anormal de células da mama, que formam um tumor com potencial de invadir outros órgãos. A pré-disposição genética está entre um dos fatores de risco para o surgimento da patologia, juntamente com obesidade, consumo de bebidas alcoólicas, tabagismo, exposição frequente a radiação ionizante (Raio X), entre outros.

Os sinais e sintomas mais presentes são a retração ou abaulamento na pele ou no mamilo, nódulos na axila, pele avermelhada, alterações na pele como casca de laranja e secreção saindo do mamilo. $O$ diagnóstico pode ser realizado através da ultrassonografia, mamografia, ressonância magnética e exames laboratoriais com marcadores tumorais cintilografia, biopsias, exames citopatológico e histopatológico (GONÇALVES LLC, et al., 2010; BERNARDES NB, et al., 2019).

Sabe-se que o tratamento para este tipo de câncer envolve uma abordagem múltipla incluindo, a radioterapia como forma de tratamento local, tratamento medicamentoso sistêmico através da quimioterapia, hormonioterapia, e a cirurgia de mastectomia. A escolha do tratamento depende da avaliação individual e criteriosa de cada caso, além da análise de alguns parâmetros como: características do tumor, da paciente e da fase em que é diagnosticada a doença (BARBOSA JAN, et al,2013)

O tratamento cirúrgico pode desencadear uma série de sequelas como dor, redução funcional homolateral a cirurgia, diminuição da amplitude de movimento, subtração da força muscular dos músculos da cintura escapular, alteração de sensibilidade, aumento de volume do segmento, parestesias e paresias. Atualmente a fisioterapia dispõe de vários recursos terapêuticos que atuam na prevenção e reabilitação das complicações bem como nas limitações funcionais; dentre esses recursos, encontra-se a cinesioterapia (VIEIRA RAC, et al, 2016).

Baracho E (2018), afirma que as técnicas de alongamento, mobilização cicatricial, pompagem, massoterapia e cinesioterapia tem o objetivo de recuperar as limitações através da melhora da força muscular e da promoção da redução de edema. Desta forma Ferreira ACM e Martins HO (2013), acreditam que a massoterapia é um conjunto de procedimentos manipulativos que tem como objetivo a estimulação circulatória e a mobilidade dos tecidos e segmentos. Santos LDFS e Pereira MCA (2016), abordam a pompagem com o intuito de promover relaxamento por meio de um alongamento lento e progressivo.

Dalvi AR, et al. (2010) aborda que a cinesioterapia dispõe de exercícios que reduzem alterações osteomioarticulares, respiratórias e circulatórias. Tortora GJ e Grabowski SR (2002), afirmam que o alongamento visa expandir o comprimento muscular permitido pela morfologia, o que pode vir a proporcionar relaxamento do mesmo. Borcard MAM (2009) aborda que a mobilização cicatricial tem via de ação através da presença de tecido cicatricial entre tecidos sadios, o que ocasiona uma adesão que compromete a mobilidade e função da área afetada. Diante do exposto, de que forma os recursos fisioterapêuticos pode contribuir na reabilitação de mulheres pós mastectomizadas?

A relevância da pesquisa dá-se pelo crescente índice de mulheres acometidas pela doença e as complicações motoras advindaspós-mastectomia onde os recursos fisioterapêuticos vêm se tornando cada vez mais utilizados por serem técnicas de fácil acesso, não farmacológicos, baixo custo e sem efeitos colaterais. Desta forma espera-se que o presente estudo venha contribuir no meio social, acadêmico e profissional, levando maiores informações acerca do assunto abordado. Neste sentido, o objetivo deste trabalho é discutir as contribuições dos princiapais recursos fisioterapêuticos na reabilitação de mulheres pós mastectomizadas. 


\section{MÉTODOS}

O presente artigo se caracteriza como uma revisão bibliográfica, sendo usado o método dedutivo, caracterizado como qualitativo, descritivo e exploratório.

A coleta de material foi realizada em bases eletrônicas de dados, tais como: Scielo, BVS, Pubmed, Pedro e Google Acadêmico. Além destes, também foi utilizada a Biblioteca da Faculdade Regional de Alagoinhas - UNIRB (FARAL) e títulos adquiridos através de indicações de profissionais fisioterapeutas. Para a realização do trabalho foram utilizadas as seguintes palavras chaves, previamente pesquisadas nos descritores em ciências da saúde (DESC): "Fisioterapia", "Mastectomia", "Saúde da Mulher". Como critério de inclusão foram aceitos artigos em idioma português, inglês e espanhol, com um horizonte temporal de 2009 até novembro de 2019. Como critério de exclusão, não foram utilizados os demais artigos que não se enquadraram nos itens de inclusão, com exceção dos livros de autoria de Kisner C e Colby LA (2005) e Totora GJ e Grabowski SR (2002), diante do fato que essas são as edições mais recentes publicadas.

\section{RESULTADOS E DISCUSSÃO}

Para a construção deste estudo foram encontrados 9 artigos, onde 5 foram selecionados para a formação do presente trabalho e 4 excluídos por não se encaixarem nos requisitos de inclusão.

Quadro 1 - Resultados encontrados nos artigos selecionados

\begin{tabular}{|c|c|c|}
\hline Autor/Ano & Obejtivo & Principais Resultados \\
\hline $\begin{array}{c}\text { Moreira F e Pivetta HMF } \\
\text { (2012) }\end{array}$ & $\begin{array}{l}\text { Comparar os efeitos da cinesioterapia } \\
\text { e da massoterapia na funcionalidade e } \\
\text { na força muscular respiratória em } \\
\text { pacientes mastectomizadas }\end{array}$ & $\begin{array}{l}\text { A cinesioterapia e a massoterapia } \\
\text { apresentou melhora na maioria dos } \\
\text { movimentos realizados pela } \\
\text { articulação do ombro e na } \\
\text { otimização da respiração }\end{array}$ \\
\hline Rett MT, et al. (2012) & $\begin{array}{c}\text { Comparar a ADM e a intensidade da } \\
\text { algia no MS homolateral a cirurgia } \\
\text { antes, durante e após o protocolo } \\
\text { cinesioterapêutico }\end{array}$ & $\begin{array}{l}\text { Cinesioterapia com a utilização de } \\
\text { alongamentos foram eficazes na } \\
\text { redução da dor e no aumento } \\
\text { significativo da ADM }\end{array}$ \\
\hline Tacani PM. et al. (2013) & $\begin{array}{c}\text { Verificar a atuação da fisioterapia } \\
\text { voltada a ADM e ao volume do MS em } \\
\text { mulheres pós mastectomia }\end{array}$ & $\begin{array}{l}\text { A utilização da cinesioterapia, } \\
\text { alongamentos e massoterapia } \\
\text { influenciou positivamente no } \\
\text { aumento da ADM do ombro. }\end{array}$ \\
\hline $\begin{array}{l}\text { Jeronimo AFA, et al. } \\
\qquad(2013)\end{array}$ & $\begin{array}{l}\text { Investigar os efeitos da cinesioterapia } \\
\text { no ganho de força muscular e na ADM } \\
\text { em pacientes pós cirúrgicas }\end{array}$ & $\begin{array}{l}\text { Foram utilizados a cinesioterapia, e } \\
\text { exercícios respiratórios, onde } \\
\text { proporcionaram melhora } \\
\text { significativa da força muscular e da } \\
\text { ADM, bem como da respiração. }\end{array}$ \\
\hline Nava LP, et al. (2016) & $\begin{array}{l}\text { Verificar a influência do protocolo } \\
\text { fisioterapêutico na funcionalidade e na } \\
\text { qualidade de vida de mulheres pós } \\
\text { mastectomizadas }\end{array}$ & $\begin{array}{l}\text { Mobilização cicatricial, alongamento } \\
\text { e a cinesioterapia mostraram-se } \\
\text { eficazes para o aumento da ADM. }\end{array}$ \\
\hline
\end{tabular}

Fonte: Sá LTS, et al. (2020) 
No trabalho de Lahoz MA, et al. (2010) afirma que a mastectomia afeta de forma negativa na qualidade de vida das mulheres que foram submetidas a este procedimento, de modo que ocorre a redução da amplitude de movimento (ADM), da força muscular, presença de algia relacionadas aos membros superiores (MMSS) afetados. Desta maneira Rett MT, et al. (2012) destaca a importância da inicialização da intervenção da forma mais breve possível, dando destaque para a cinesioterapia que aumentou consideravelmente a ADM do MS afetado e subtraiu o quadro álgico do MS homolateral à cirurgia.

Segundo Pacheco MN, et al. (2011), a fisioterapia utiliza-se de recursos como mobilização articular, alongamentos, cinesioterapia, drenagem linfática, enfaixamento funcional, eletroterapia, massoterapia para compor o tratamento fisioterapêutico das mulheres mastectomizadas visando a redução de complicações proporcionadas pela intervenção cirúrgica e beneficiar a volta as atividades de vida diária (AVD's), a qualidade de vida.

Pacheco MN, et al. (2011), ainda destaca que os melhores resultados podem ser observados no momento em que ocorre a intervenção precoce da equipe de fisioterapia no pós-operatório, bem como quando o tratamento ainda é iniciado na fase pré-operatória, afirmando que mulheres que foram encaminhadas posteriormente à fisioterapia obtiveram quadro álgico e linfedema, o quê avigora a eficácia da intervenção precoce. Conseguinte a isto, quanto mais breve iniciado o tratamento, mais rápido a mulher irá responder ao mesmo.

Moreira F e Pivetta HMF (2012) entram em acordo com Rett MT, et al. (2012) quando afirmam que a cinesioterapia através do alongamento dos músculos de cervical e de membros superiores tem efeitos positivos para a mulher pós mastectomizada. Tendo isto em vista, Alencar TAMD e Matias KFS (2010) explanam que o funcionamento do alongamento ocorre através do aumento do comprimento das fibras musculares contribuindo para a expansão da flexibilidade articular, ou seja, ganho de amplitude de movimento.

Rett MT, et al. (2012) em congruência com Nava LP, et al. (2016) juntamente com Moreira F e Pivetta HMF (2012) alcançaram resultados positivos a respeito da funcionalidade e do aumento da amplitude de movimento do ombro homolateral à cirurgia, através do alongamento, devido ao fato de que, segundo Tortora GJ e Grabowski SR (2002), os sarcômeros da fibra muscular são expandidos até o máximo comprimento permitido pela morfologia, desta forma, a zona de sobreposição é reduzida, como resultado a tensão que pode ser produzida pela fibra é significativamente diminuída.

Tacani PM, et al. (2013) corrobora com Moreira F e Pivetta HMF (2012) quando optam pela utilização da massoterapia como um recurso para diminuição do quadro álgico. Abreu MF, et al. (2012) afirma que este efeito é possível em prol do fato de que a massoterapia estimula o crescimento do suplemento de sangue e consequentemente a recepção de oxigênio, o que por sua vez acarreta no alívio das algias, diminuição do estresse e maior controle acerca dos espasmos musculares.

O alcance de resultados satisfatórios com a utilização da massoterapia, encontrados por Moreira $\mathrm{F}$ e Pivetta HMF (2012) e Tacani PM, et al. (2013), tornou-se possível tendo em vista que Ferreira ACM e Martins HO (2013) apresentam que o recurso otimiza a oxigenação, nutrição e chegada de substancias produzidas pelo organismo que acarretam melhoras ao quadro álgico em congruência com a otimização da circulação sanguínea, bem como da mobilidade que proporciona uma maior flexibilidade dos tecidos.

Tacani PM, et al. (2013) entram em harmonia com Rett MT, et al. (2012) quando usam de exercícios ativos para o ganho de amplitude de movimento e de força muscular. Desta forma, a cinesioterapia realizada pelo uso de exercícios ou movimentos terapêuticos como flexo-extensão, e rotação interna e externa, proporciona a prevenção, manutenção, correção e recuperação da função e da força muscular da articulação comprometida, mantendo a integridade de força, mobilidade, flexibilidade e coordenação motora (KISNER C \& COLBY LA, 2005).

Jeronimo AFA, et al. (2013) utilizou da cinesioterapia, alcançando melhora da força muscular e da amplitude de movimento, bem como, recuperação da funcionalidade da articulação glenoumeral. Conforme Baracho E (2018) este resultado se tornou possível, pois a cinesioterapia oferece uma vida de ação para a diminuição do linfedema quando a contração muscular favorece a ação do sistema linfático aumentando a absorção e potencialização da circulação venosa. 
Para Quinto SMG e Meija DPM (2012) os movimentos cinesiológicos colaboram para a integração do membro acometido com o restante do corpo, possibilitando além da volta a rotina a aceitação do corpo. Onde os autores enfatizam que o uso da cinesioterapia em conjunto com a drenagem linfática proporciona um rápido alivio das alterações causadas pelo linfedema, tendo uma função primordial no pos operatório de mastectomia, ainda sendo crucial para a manutenção do membro superior (MS) acometido.

Quinto SMG HL e Meija DPM (2012) ainda observaram que obtém inúmeros benefícios da drenagem linfática manual (DLM) associada à cinesioterapia no tratamento de mulheres pós mastectomizadas com linfedema subtraindo ao mesmo com a redução do edema do membro afetado, influenciando positivamente na amplitude de movimento.

Nava LP, et al. (2016), fez uso da mobilização cicatricial para obter resultados positivos no tratamento. Borcard MAM (2009) afirma que o alcance dos resultados foi possível diante do fato que quando há tecido cicatricial entre tecidos sadios ocorre à restrição da mobilidade entre eles, o que ocasiona uma adesão que compromete a mobilidade e função da área afetada.

Segundo Bocard MAM (2009), a liberação tecidual funcional, provoca um tensionamento constante e prolongado que organiza a disposição do colágeno oferecendo mais elasticidade ao tecido, prevenindo e tratando fibroses e aderências, sendo considerada essa a técnica mais efetiva e rápida para o tratamento dessas lesões.

Rett MT, et al. (2012), realizou um estudo onde durante o tratamento utilizou a mobilização cicatricial para melhora de amplitude de movimento. Segundo Nascimento SL et al. (2012), os resultados tonaram-se possíveis de serem contemplados tendo em vista que a mobilização da cicatriz proporciona o alongamento do tecido conjuntivo, diminuição de aderências e alivio da sensibilidade, o que por sua vez otimiza 0 movimento fisiológico e subtrai o quadro álgico, além de contribuir para a evolução da qualidade de vida da paciente.

Padro Junior JRA, et al. (2017) realizou um estudo onde utilizou da mobilização para ganho da amplitude do movimento e redução do quadro álgico das disfunções advindas do processo de mastectomia, onde o mesmo conseguiu obter resultados positivos aumentando o arco do movimento e como conseguinte a funcionalidade do membro acometido e a redução do quadro álgico. Ainda segundo Bocard MAM (2009), a influência positiva é possível diante do fato que ao realizar a mobilização reorganizam-se as fibras do tecido conjuntivo.

Através da mobilização cicatricial Nava LP, et al. (2016) e Rett MT, et al. (2012) alcançaram resultados positivos, prestando evidencia para o aumento da amplitude de movimento. Tendo em vista que segundo Petter GN, et al. (2015), afirma que após a mastectomia surgem retrações cicatriciais que limitam a amplitude de movimento, reduzindo conseguintemente a realização de atividades básicas da vida diária, como por exemplo pentear o cabelo.

Petter GN, et al. (2015) aborda que a liberação miofascial possibilita a diminuição do quadro álgico e otimiza a funcionalidade do membro superior acometido em mulheres mastectomizadas, mesmo quando usada de forma singular, por isto os autores destacam a terapia manual como crucial no processo de tratamento visando evitar limitações derivadas das retrações miofasciais como sobrecargas biomecânicas, quadro álgico e subtração da funcionalidade.

Zanon DS, et al. (2017) realizou um estudo onde não alcançou resultados significantes com o uso de massagem cicatricial em mulheres pós mastectomizadas. Entretanto, o mesmo estudo destaca a possibilidade de encontro deste resultado decorrente da baixa amostra e do curto período de tempo que foi disponibilizado para o estudo, solicitando que ocorra mais estudos que possam vir a esclarecer os reais efeitos com uma amostra maior, bem como com um tempo adequado.

Martins JF, et al. (2012) acorda com Tacani PM, et al. (2013), quando relata a obtenção de resultados positivos com a utilização da pompagem em pacientes pós mastectomia. Onde, segundo Silva LMV e Maia FB (2018), a técnica proporciona a mulher mais qualidade na realização de movimentos bem como aumenta a amplitude de movimentos e reduz a algia, permite que o paciente adquira maior percepção do segmento, 
acarretando na compreensão do dinamismo que é necessário para o desempenho da função. A consciência de que o segmento comprometido faz parte da globalidade corporal oferece um prognostico positivo ao tratamento.

A pompagem é uma técnica da terapia manual onde oferece relaxamento muscular, otimização da circulação e regeneração articular, sendo ela dívida em três etapas: o tensionamento, manutenção da tensão e tempo de retorno. Durante a aplicação da técnica o terapeuta não deve perder o contato com a pele do paciente, ultrapassar os limites fisiológicos do tecido e estar atento ao fato de que a manobra deve ser realizada com o acompanhamento de um adequado padrão respiratório (SANTOS JUNIOR FFU, 2016).

Antunes MD, et al. (2017) fez uso da pompagem para melhora da redução do quadro álgico em mulheres, e obteve resultados positivos na qualidade de vida das mesmas. Conforme Santos LDFS e Pereira MCA (2016) a pompagem promove o relaxamento das fáscias por meio de um alongamento lento, regular e progressivo, o que acarreta em ganho de amplitude de movimento, bem como uma redução significativa do quadro álgico.

Em um estudo realizado por Sales TOP, et al. (2013), as pacientes que obtiveram acesso a terapia através do uso da técnica de pompagem, apresentaram um ganho significativo, tendo em vista que essas relaxavam mais durante 0 tratamento em comparação às mulheres que utilizaram a cinesioterapia. Enquanto o grupo que teve acesso às duas técnicas associadas demostrou melhores resultados visto que uma técnica potencializa a efetividade da outra.

Pujol-Blaya V, et al. (2019), em seu estudo, realizou um tratamento através do uso de drenagem linfática manual juntamente com um sistema de compressão, onde notabilizou-se que houve reduções significativas no excesso de volume do edema presente. Luz ND e Lima ACG (2011), afirmam que a atuação dos recursos tem via de ação nos vasos linfáticos, incitando a reabsorção e a condução do acúmulo de líquido edemaciado para áreas não afetadas e estimulando o desenvolvimento das vias colaterais de drenagem com o objetivo de controlar a expansão do edema a longo prazo.

No artigo de autoria de Quinto SMG e Meija DPM (2012) foi abordado que a drenagem linfática manual (DLM) mostrou-se eficiente no tratamento mesmo quando usada de forma singular, entretanto quando utilizada em conjunto com outras técnicas tornou-se possível obter melhores resultados, tendo em vista que um recurso potencializa os efeitos um do outro e vice-versa.

A intervenção da fisioterapia com o uso da drenagem linfática, enfaixamento compressivo, contenção elástica e cinesioterapia, proporcionam benefícios na prevenção e no tratamento de linfedema. Ainda dando destaque a efetividade quando realizada da forma mais breve possível para prevenir as limitações acarretadas pela pós-mastectomia, sendo elas a aderência cicatricial, retrações, fibrose, algia na incisão, alterações da sensibilidade, alterações posturais, linfedema e a diminuição da amplitude de movimento. É importante destacar que a mulher que realiza o tratamento fisioterapêutico reduz o tempo de recuperação e volta o mais breve possível às atividades diárias e ocupacionais (QUINTO SMG e MEIJA DPM, 2012).

Luz ND e Lima ACG (2011) em sua pesquisa afirmam que a bandagem ou enfaixamento compressivo, é utilizado para manter e incrementar os efeitos da drenagem linfática manual, aumentando o fluxo linfático e prevenindo um novo acumulo de fluidos após a drenagem. Deve ser funcional com pressão maior em nível distal sendo realizada na primeira fase da fisioterapia complexa descongestiva, sempre após a drenagem linfática.

Um estudo realizado por Tacani PM, et al. (2013) expõe que a fisioterapia descongestiva obteve resultados positivos no tratamento de linfedema de membros superiores após mastectomia, sendo utilizados de forma conjunta técnicas manuais (drenagem linfática, massagem, pompagem, vácuo terapia e massagem cicatricial), eletroterapia (alta frequência e TENS), cinesioterapia (exercícios miolifocinéticos, ativo-assistido e flexibilidade) e terapia compressiva (braçadeira elástica). Desta forma, neste trabalho tornou-se possível evidenciar a redução do linfedema, do quadro álgico e da alteração da sensibilidade.

Ainda segundo Luz ND e Lima ACG (2011), em seu artigo afirma que a fisioterapia complexa descongestiva (FCD) possui recursos como a drenagem linfática manual, vestuário de compressão, 
bandagens, meticulosa higiene da pele e exercícios terapêuticos, obtendo duas fases: onde a primeira é caracterizada por um tratamento diário, aplicado por um espaço de tempo de duas a quatro semanas, sendo encerrada ao alcançar a máxima redução do linfedema. Enquanto na segunda fase é caracterizada como de manutenção, dando destaque a terapia de compressão, com a finalidade de manter os efeitos positivos logrados na primeira fase.

A fisioterapia oferece a possibilidade de tratar o linfedema otimizando e mantendo a funcionalidade da circulação linfática, reduzindo o risco de recidiva e de infecções. Tendo isto em vista, os autores afirmam que os recursos fisioterapêuticos são a forma mais eficiente de tratamento para a mastectomia onde obtém crucial papel na manutenção cinético-funcional da cintura escapular e recuperação dos movimentos dos MMSS (LUZ ND e LIMA ACG, 2011)

\section{CONSIDERAÇÕES FINAIS}

As técnicas fisioterapêuticas utilizadas para a construção deste estudo proporcionaram resultados positivos, oferecendo a estas pacientes a melhora no quadro álgico e na funcionalidade necessária para o retorno as suas atividades. Durante a construção deste artigo houve dificuldades para encontrar trabalhos que fossem realizados por profissionais atuantes da área de fisioterapia, desta maneira, devem ser incentivados que ocorram mais estudos voltados para a atuação dos recursos fisioterapêuticos em mulheres mastectomizadas para a retirada de carcinoma mamário. Além destas, ocorreram obstáculos para evidenciar a via de ação das técnicas dentro do horizonte temporal de 10 anos, destacando-se a modalidade do alongamento muscular.

\section{REFERÊNCIAS}

1. ABREU MF, et al. Os Efeitos da massoterapia sobre o estresse físico e psicológico. Revista Científica da Faculdade de Educação e Meio Ambiente, 2012; 3: 101-105.

2. ALENCAR TAMD, MATIAS, KFS. Princípios fisiológicos do aquecimento e alongamento muscular na atividade esportiva. Rev Bras Med Esporte, 2010; 16(3): 230-234.

3. ANTUNES MD, et al. Análise comparativa dos efeitos da massoterapia e pompage cervical na dor e qualidade de vida em mulheres. ConScientiae Saúde, 2017: 16(1): 109-115.

4. BARACHO E. Fisioterapia aplicada à Saúde da Mulher. 6oe. Rio de Janeiro. Guanabara Koogan. 2018.

5. BARBOSA JAN, et al. Avaliação da postura corporal em mulheres com câncer de mama. Revista Brasileira de Ginecologia e Obstetrícia, 2013; 35(5): 215-220.

6. BAVARESCO GZ, et al. O fisioterapeuta como profissional de suporte à parturiente. Ciência \& Saúde Coletiva, 2011; 16: 3259-3266.

7. BERNARDES NB, et al. Câncer de Mama $X$ Diagnóstico/Breast Cancer $X$ Diagnosis. Id online revista multidisciplinar e de psicologia, 2019: 13(44): 877-885.

8. BORCARD MAM. Abordagem cinesioterapêutica em pós-cirúrgico de câncer de mama do tipo quadrantectomia. Fisioterapia Brasil, 2009; 10(4): 290-293.

9. BRASIL, Ministério da saúde. Estimativas da incidência e mortalidade por câncer. Rio de Janeiro. 2018.

10. DALVI AR, et al. Beneficios da cinesioterapia a partir do segundo trimestre gestacional. Revista Saude e Pesquisa, 2010; 3(1): 47-51.

11. FERREIRA ACM, MARTINS HO. Movimentação passiva continua: Conceito e aplicação. Caderno de Estudos Tecnologicos, 2013; 1(1): 177-188.

12. GUSMÃO C. Drenagem Linfática Manual: Método Dr. Vodder. São Paulo: Atheneu, 2010.

13. GONÇALVES LLC, et al. Fatores de risco para câncer de mama em mulheres assistidas em ambulatório de oncologia. Rev. enferm, 2010; 18(3):468-72.

14. INCA incidência de câncer no Brasil. Estimativa. Revista Brasileira de Cancerologia, 2014; 60(1): 63-64.

15. JERÔNIMO AFA, et al. Efeitos da cinesioterapia na força muscular e amplitude de movimento em pacientes mastectomizadas. Suplemento Especial, p. 5, 2013.

16. KISNER C, COLBY LA. Exercícios Terapêuticos Fundamentos e Técnicas. 4ª Ed. São Paulo: Manole, 2005.

17. LAHOZ MA, et al. Capacidade funcional e qualidade de vida em mulheres pós-mastectomizadas. Revista Brasileira de Cancerologia, 2010; 56(4): 423-430.

18. LUZ ND, LIMA ACG. Physical therapy resource in post-mastectomy lymphedema: a review of the literature. Fisioterapia em Movimento, 2011;24(1):191-200.

19. MAGNO RBC. Bases reabilitativas de fisioterapia no câncer de mama. 68 f. Monografia (Trabalho de Conclusão de Curso) - Universidade Veiga de Almeida, Rio de Janeiro, 2009.

20. MOREIRA F, PIVETTA HMF. Efeitos da cinesioterapia e massoterapia sobre a funcionalidade do ombro e força muscular respiratória de mulheres mastectomizadas. Fisioterapia Brasil, 2012; 13(4): 250-255. 
21. MARTINS JF, et al. Terapia manual na restauração da amplitude articular do ombro em mulher mastectomizada. Fisioter. Bras, 2012.

22. NASCIMENTO SL, et al. Complicações e condutas fisioterapêuticas após cirurgia por câncer de mama: estudo retrospectivo. Fisioterapia e Pesquisa, 2012.

23. NAVA LP, et al. Funcionalidade de membro superior e qualidade de vida de mulheres com câncer de mama submetidas a tratamento fisioterapêutico. Revista de Atenção à Saúde (antiga Rev. Bras. Ciên. Saúde), 2016; 14(48): 21-26.

24. PACHECO MN, et al. Fisioterapia para o tratamento do linfedema no pós-operatório de mastectomia: revisão de literatura. Revista da Faculdade de Ciências Médicas de Sorocaba, 2011, 13(4): 4-7.

25. PADRO JUNIOR JRA, et al. Efeito imediato da técnica de mobilização nas interfaces fasciais profundas da região peitoral em pacientes submetidas à mastectomia. Fisioterapia Brasil, 2017, 18(2): 180-188.

26. PETTER GN, et al. Efeitos da liberação miofascial sobre a funcionalidade e a dor em mulheres mastectomizadas. Fisioterapia Brasil, 2015, 16(3): 202-206.

27. PUJOL-BLAYA V, et al. Effectiveness of a precast adjustable compression system compared to multilayered compression bandages in the treatment of breast cancer-related lymphoedema: a randomized, single-blind clinical trial.Clin Rehabil, 2019; 33 (4): 631-641.

28. QUINTO SMG, MEJIA DPM. Benefícios da fisioterapia no tratamento de linfedema pós-mastectomia radical: uma revisão literária. 2012.

29. RETT MT, et al. A cinesioterapia reduz a dor no membro superior de mulheres submetidas à mastectomia ou quadrantectomia. Rev Dor. São Paulo, 2012; 13(3): 201-207.

30. SALES TOP, et al. Fisioterapia oncológica em pacientes mastectomizadas através de pompages e técnicas cinesioterápicas. Revista interfaces: saúde, humanas e tecnologia, 2013, 1(1).

31. SILVA LMV, MAIA FB. Contribuições da Pompage na prática da terapia ocupacional na reabilitação física/Contributions of Pompage in the practice of occupational therapy in physical rehabilitation. Revista Interinstitucional Brasileira de Terapia Ocupacional-REVISBRATO, 2018; 2(3): 654-667.

32. SOUZA RL, MEJIA DPM. A drenagem linfática-técnica vodder associada a cinesioterapia em pós-operatório imediato de mastectomia para a manutenção da funcionalidade do ombro. 2015.

33. SOUZA APK, RAMOS DJS. Fisioterapia e humanização do parto: Uma analise a partir de documentos oficiais da saúde. Revista Fisioterapia Reabilitação, 2017; 1(1): 11-23.

34. SANTOS JUNIOR FFU. Atividade elétrica muscular em portadores de disfunções temporomandibulares: uma revisão sistemática. Neuroc Psicol. 2016;11(2):89-97.

35. SANTOS LDFS, PEREIRA MCA. A efetividade da terapia manual no tratamento de disfunções temporomandibulares (DTM): uma revisão da literatura. Rev Bras Ciên Saúde. 2016;14(49):727.

36. TACANI PM, et al. Fisioterapia em grupo na reabilitação funcional dos membros superiores de mulheres pósmastectomia. Ter Man, 2013; 51(11): 1-6.

37. TORTORA GJ, GRABOWSKI SR. Princípios de Anatomia e Fisiologia. 9a ed. Rio de Janeiro: Guanabara Koogan, 2002.

38. VIEIRA, RAC et al. Instrumentos de avaliação quantitativa e qualitativa das sequelas relacionadas ao tratamento do câncer de mama. RevBras Mastologia, 2016; 26(3): 126-32.

39. VISCONE AC, et al. Efeito da cinesioterapia na força muscular de mulheres mastectomizadas. In: Colloquium Vitae. p. 163-167,2013.

40. ZANON DS, et al. Efeito da massagem miofascial sobre a dor e a propriocepção pós-mastectomia radical. Revista de Terapia Ocupacional da Universidade de São Paulo, 2017, 28(1): 115-121. 